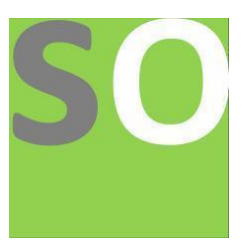

Article title: A Sentinel based agriculture monitoring scheme for the control of the CAP and food security

Authors: Antonios Koutroumpas[1], Vasileios Sitokonstantinou[2], Thanassis Drivas[3], Alkiviadis Koukos[4], Vassilia Karathanassi[5], Haris Kontoes[6], loannis Papoutsis[7]

Affiliations: Institute for Space Applications and Remote Sensing, National Observatory of Athens, I.Metaxa and Vas. Pavlou St, Penteli, 15236 Athens, Greece[1], Laboratory of Remote Sensing, National Technical University of Athens, Heroon Polytechniou 9, 15780 Zografou, Greece[2]

Orcid ids: 0000-0001-5017-9975[1]

Contact e-mail: antonkout@gmail.com

License information: This work has been published open access under Creative Commons Attribution License http://creativecommons.org/licenses/by/4.0/, which permits unrestricted use, distribution, and reproduction in any medium, provided the original work is properly cited. Conditions, terms of use and publishing policy can be found at https://www.scienceopen.com/.

Preprint statement: This article is a preprint and has not been peer-reviewed, under consideration and submitted to ScienceOpen Preprints for open peer review.

DOI: 10.14293/S2199-1006.1.SOR-.PPHMESU.v1

Preprint first posted online: 07 July 2021

Keywords: remote-sensing, CAP, phenology-extraction 


\title{
A Sentinel based agriculture monitoring scheme for the control of the CAP and food security
}

\author{
Vasileios Sitokonstantinou*a, Antonios Koutroumpas ${ }^{\mathrm{a}}$, Thanassis Drivas ${ }^{\mathrm{a}}$, \\ Alkiviadis Koukos ${ }^{\mathrm{a}}$, Vassilia Karathanassi ${ }^{\mathrm{b}}$, Haris Kontoes ${ }^{\mathrm{a}}$, Ioannis \\ Papoutsis ${ }^{\mathrm{a}}$, \\ ${ }^{a}$ Institute for Space Applications and Remote Sensing, National Observatory \\ of Athens, I.Metaxa and Vas. Pavlou St, Penteli, 15236 Athens, Greece. \\ ${ }^{\mathrm{b}}$ Laboratory of Remote Sensing, National Technical University of Athens, \\ Heroon Polytechniou 9, 15780 Zografou, Greece
}

\begin{abstract}
Effective and efficient control of the agrarian obligations imposed by the Common Agricultural Policy (CAP) and the high-level decision making for national and global food security, requires systematic and timely monitoring of the agricultural land. In this study we focus on rice paddy monitoring in South Korea to ultimately deliver food security related information. Food security monitoring demands knowledge at large scales to allow for decision making at the highest level. In this work, we monitor the growth of rice using the TIMESAT solution on a time-series of Normalized Difference Vegetation Index (NDVI), extracting useful metrics with reference to the phenological phases of the crop, but also biomass and yield indicators. TIMESAT requires user provided parameters to define the start and the end of season to then compute the relevant metrics. In order to automate this procedure, the vegetation indices Normalized Difference Water Index (NDWI) and Plant Senescence Reflectance Index (PSRI) are used to develop a data based parameter tuning for TIMESAT.
\end{abstract}

Keywords: Sentinel Missions, Regression Fitting; Phenology; Food Security; Yield Estimation 


\section{INTRODUCTION}

\subsection{Common Agricultural Policy and Food Security}

Climate change and the expected increase in the global population have shaped new requirements to guarantee food security ${ }^{1}$. In that respect, the European Union (EU) has set priorities to increase its agricultural productivity under environmentally friendly practices, investing over $40 \%$ of its yearly budget ${ }^{2}$. The new Common Agricultural Policy (CAP), the policy framework of EU, aims to effectively integrate pertinent emerging Earth Observation (EO) and ICT technologies into the daily business of farmers and policy implementation bodies. The need for increased productivity brings precision agriculture in the foreground to play a substantial role in satisfying the requirements for food, while ensuring environmentally sustainable land-use ${ }^{4}$. Precision agriculture is a technology that improves the agricultural management by collecting geo-referenced data, such as soil properties, weather data and crop status indicators at the parcel level, having a strong potential to assist in CAP's regulations monitoring and environmental impact evaluation ${ }^{5}$. Within this framework, smart farming services, such as precise knowledge of crop growth stages and timely yield estimation, support farmers to optimize the management of fertilizers and irrigation, while minimizing the impact on natural resources, meeting the environmental goals of the CAP.

The recently launched Sentinel missions, opened new opportunities in terrestrial monitoring and therefore in agriculture applications, providing open access EO data with high spatial and temporal resolution. The availability of free, high quality and frequent EO data, covering large areas have proved ideal to address regional and global food security needs (e.g., GEOCRADLE, GEOGLAM, e-shape). Finally, it could be argued that Sentinels' effectiveness has attracted great attention in agricultural monitoring activities and specifically within the context of the CAP $(\underline{\text { RECAP }}, \underline{\text { Sen } 4 C A P})^{8-9}$.

\subsection{Crop Classification Approaches}

Crop classification stands at the foundation of the majority of EO based agriculture monitoring systems. Recent publications have proposed different machine and deep learning approaches, utilizing time-series of multispectral imagery. Non-parametric supervised classifiers such as Support Vector Machines (SVM) and ensemble decision trees, such as Random Forest (RF), have been successfully implemented in crop classification schemes ${ }^{6,7}$. A comparative analysis, by Sitokonstantinou et al. $(2018)^{8}$, between SVM and RF, demonstrated the effectiveness of SVM for accurate crop classification and the efficiency of RF for big datasets. Deep learning has also been extensively used for crop identification. A recent publication by Mazzia et al. 
$(2020)^{9}$ used a scheme of deep learning for a pixel-based land cover and crop classification analysis. The authors utilized a Recurrent Neural Networks (RNN) in combination with a Convolutional Neural Networks (CNN) using multi-temporal Sentinel-2 imagery, achieving an overall accuracy of $96.5 \%$.

\subsection{Crop growth and yield estimation}

The knowledge of crop's status enables the effective decision making and planning of agricultural practices, such as the appropriate fertilization and irrigation operations, to maximize the yield ${ }^{10}$. In this context, the correlation between satellite-based features, such as vegetation indices (VIs), and biophysical parameters collected at a single date, or accumulated over some time period, is investigated. Published studies, like Lambert et al $(2018)^{11}$, took advantage of Landsat and Sentinel-2 data to estimate crop yield using regression fitting of smoothed VI curves.

Considering the drawbacks of satellite imagery due to cloud cover, EO indices can only reveal coarse growth stages, where short-interval growth stages can be effectively identified using ancillary climate and meteorological data (e.g. air temperature). Since crops grow via the absorption of heat, this can be quantitatively measured by air temperature accumulation with the construction of Accumulated Growing Degree Days ${ }^{12}$. In addition, Duchemin et al (2006) ${ }^{13}$ tailored a first approach at coupling remote sensing vegetation indices with evapotranspiration data to investigate wheat phenology and irrigation practices in Central Morocco, revealing a strong relationship between the Leaf Area Index (LAI) and the crop coefficient. Moreover, other publications approach yield estimation through the exploitation of Crop Growth Models, such as the AquaCrop ${ }^{14-16}$ model and the World Food Studies Simulation Model (WOFOST) ${ }^{17-}$ ${ }^{18}$, in combination with satellite data, to simulate the phenology development processes and therefore predict the yield production.

Recent studies, employ more complex deep learning techniques in phenology extraction and yield estimation, utilizing EO data and Vegetation Indices ${ }^{19-22}$. Haghverdi et al $(2018)^{20}$ used Landsat 8 data, including simple ratio (SR), NDVI, green NDVI (GNDVI) and indices of greenness, wetness and soil brightness (GI, WI, SBI) as input data to an artificial neural network (ANN) in order to relate Crop Indices and field estimates and ultimately predict the yield of cotton fields. In the same context, Hulya Yalcin (2017) ${ }^{22}$ have developed a phenology recognition algorithm, using a pre-trained Convolutional Neural Network (CNN) and ground field mounted cameras to classify the phenological stages of various types of plants. 


\subsection{The proposed study}

This study suggests a fully automated pipeline for the continuous and timely monitoring of rice in South Korea, to produce yield estimation proxies and indicators. In this context, this study is as an extension of the transferable and scalable solution to address the Big Data issues associated with large-scale agriculture monitoring, as proposed by Sitokonstantinou, et al. $(2019)^{23}$. In this publication, the authors have used a High Performance Data Analytics (HPDA) environment to enable the management of long time-series of Copernicus satellite data, required for the effective and efficient monitoring of food security. The work reported herein, builds upon the published results of distributed RF rice classification, using the clustercomputing framework of Apache Spark ${ }^{23}$. The extracted rice extent for the area of interest is utilized here to subsequently extract phenological information based on the analyses of Sentinel-2 NDVI time-series. Thematic maps of phenological metrics are produced representing different stages of crop growth, such as start, peak and end of season, as well as two proxies of accumulated biomass and produced yield.

\section{DATA AND AREA OF INTEREST}

\subsection{Area of interest}

The area of interest for the present study is located at the western part of South Korea, and that is the districts of Dangjin and Seosan. These two areas represent two of the highest rice producers of the country ${ }^{24}$. The annual mean temperature in Dangjin and Seosan is $11.4^{\circ} \mathrm{C}$, while the annual precipitation is approximately $1158.7 \mathrm{~mm}^{25}$. South Korea's climate is highly affected by the Asian Monsoon. During the winter cold air masses dominate, while during the summer, warm and moist air masses reach the country from tropical regions. For this reason, the rainiest season in South Korea is during the summer, which results in scarcity of cloud free optical imagery. Figure 1 presents the area of interest for which the phenology extraction approach was implemented. 

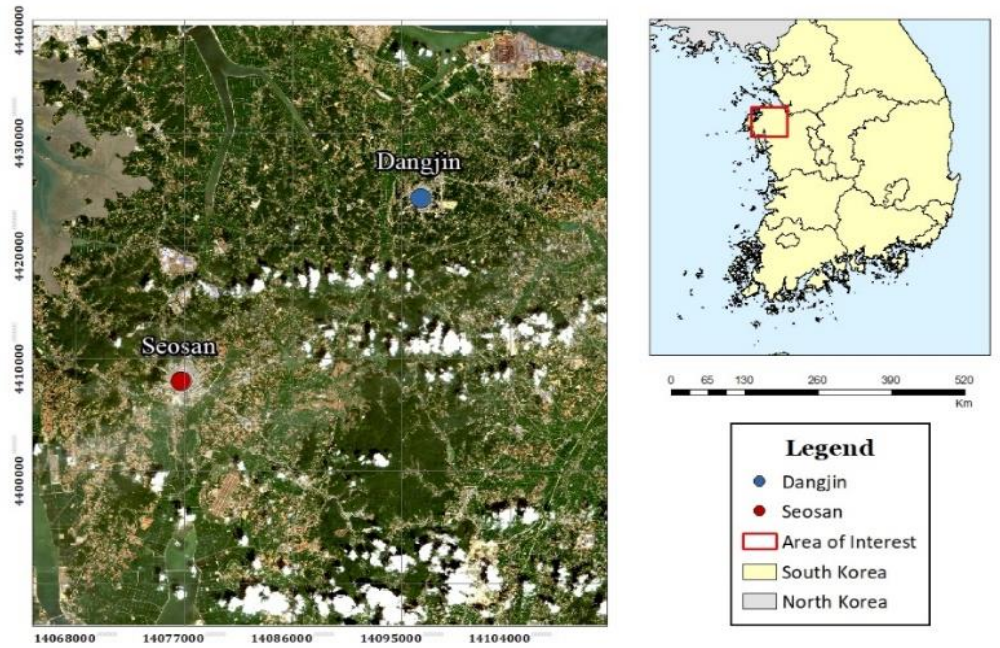

Figure 1. Area of interest located in regions of Dangjin and Seosan in South Korea.

\subsection{Data}

The input dataset for the rice classification, as described in Sitokonstantinou et al (2019) ${ }^{23}$, which was used to extract the rice extent for this study, comprises of a time-series of Sentinel1 VV backscatters, Sentinel 2 images (all bands except B01, B09, B10), and pertinent Vegetation Indices that have been widely used in crop monitoring and crop mapping applications ${ }^{26,8}$. The aforementioned feature space amounted to more than $80 \mathrm{~GB}$, which justifies then need for distributed processing, as described in the first section. The phenology extraction methodology in this study, makes use of three Sentinel-2 Vegetation Indices, including the Normalized Difference Vegetation index (NDVI), Normalized Difference Water Index (NDWI) and Plant Senescence Reflectance Index (PSRI), in order to identify the beginning, as well as the end of the crop's growth cycle. The images have been atmospherically corrected using the Sen2Cor tool and clouds have been masked out using the Sen2Cor Scene Classification product, including cloud shadows, dark pixels, high and low probability clouds, and cirrus clouds. Finally, the spatial resolution of all indices has been resampled to $10 \mathrm{~m}$ and 
amounts to several tens of gigabytes of feature space. Figure 2 illustrates how the satellite image acquisitions capture the different phenological phases in South Korea.

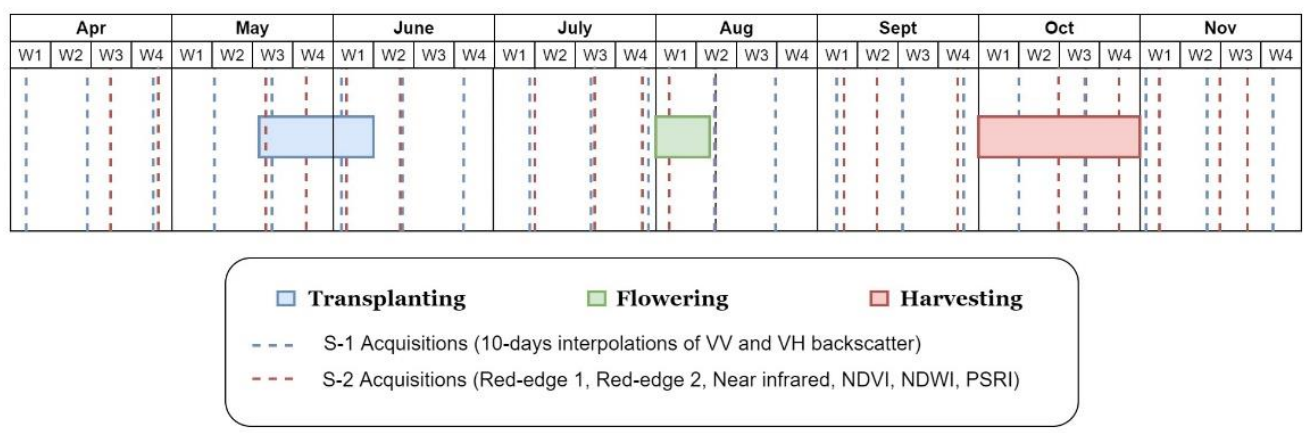

Figure 2. South Korea rice paddy growth phases and the respective Sentinel-1 and Sentinel-2 acquisitions.

\section{METHODS}

The phenology extraction methodology was implemented using TIMESAT $^{27}$, a softwarepackage that smooth satellite derived time-series data, using regression fitting. It provides three different fitting approaches, which are based on the least square fits to the upper envelope of the time-series data. In this work, Normalized Difference Vegetation Index (NDVI) time-series data were analyzed in order to obtain growth information for rice. Among the three available processing methods (Savitzky Golay, assymetric Gaussian and double logistic), the adaptive Savitzky Golay filter was used, as it captures both subtle and rapid changes in the time-series, providing a better understanding for the beginning and for the ending of the growing season. Moreover, the adaptive Savitzky Golay filter, as a form of moving average, succeeds in smoothing the NDVI curves, and at the same time fitting the time-series without reshaping the raw data After the NDVI curves are smoothed, a number of phenological metrics are computed, as shown in Table 1.

\subsection{Data Pre-Processing}

In order to analyze the NDVI curves, TIMESAT needs even spacing between the points that comprise the time-series. For this reason, several interpolation methods were investigated. 
Ultimately, the weighted average and linear interpolation methods were utilized in order to create decadal time-series from the raw data acquisitions. The weighted average interpolation method fills the fixed timestamps (decadal time-series) that fall within a ten day range of the acquisition, and then linear interpolation follows to fill any outstanding timestamps.

\subsection{TIMESAT implementation}

Initially, median filtering method is employed with multiple iterations for spike removal, as outliers may seriously degrade the final function fit and interfere with the final width and weight of the annual NDVI curve. Then the Savitzky Golay function is applied to smooth the timeseries curve.

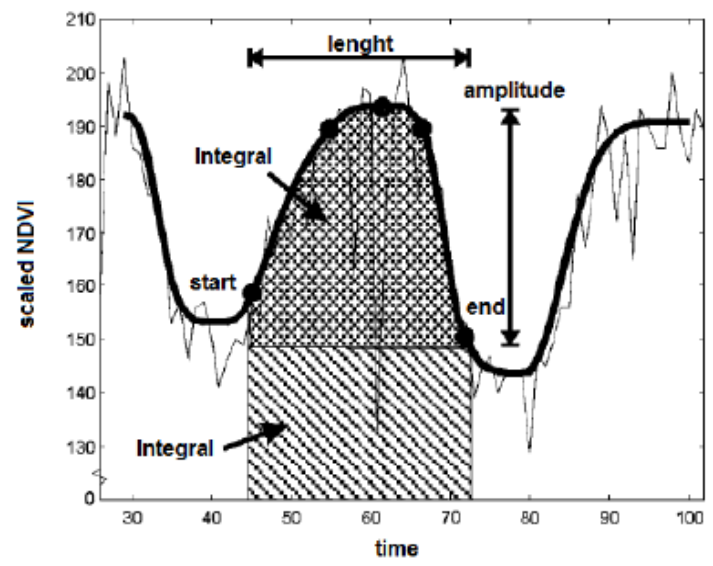

Figure 3. TIMESAT ${ }^{28}$ smoothing process and the calculated phenological metrics.

In order to extract the phenological metrics, TIMESAT requires a user-defined parameter, which describes the season start (emergence) and end (harvest). In more detail, TIMESAT requires a user specified threshold of i) the distance between the left minimum level and the maximum of the smoothed curve for the time of the start of the season (SOS) and ii) the distance between the right minimum level and the maximum for the time of the end of season (EOS). In order to automate this procedure, and create a generalized crop growth retrieval methodology, this study investigates two more vegetation indices (NDWI and PSRI), which have been preprocessed identically to the NDVI timeseries. Analyzing the time-series of NDWI and PSRI and considering the temporal cultivation practices relevant for the rice crop in South Korea, the 
SOS and EOS parameters were determined. Specifically, the transplanting (emergence) practice was identified at the intersection of NDWI and NDVI. Prior to the intersection of the curves, NDWI rises as the plots are flooded. Then NDWI drops and NDVI rises, indicating that transplanting has taken place. In the same fashion, harvesting is identified close to the intersection of PSRI and NDVI. PSRI rises, while NDVI decreases due to the yellowing of the crop. Then both NDVI and PSRI drop, indicating that harvest took place.

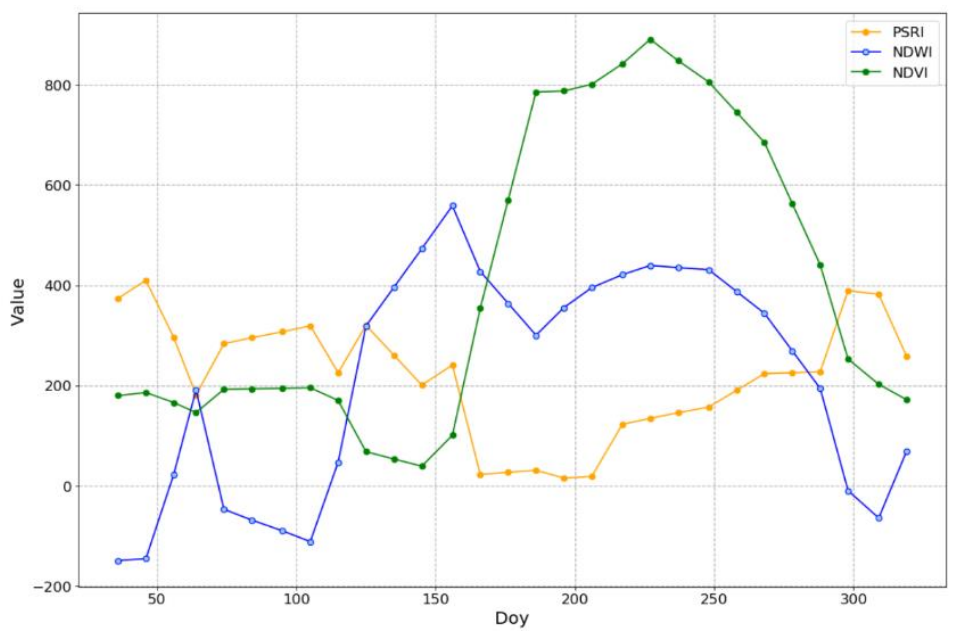

Figure 4. Interpolated time-series of NDVI, NDWI and PSRI of one parcel identifying major cultivation practices.

The products of TIMESAT are eleven phenological metrics, six of which were used in this study, as seasonality parameters. The most important of them being the start, peak and end of season, measured in Days of Year (DOY), and the rate of increase and decrease and the large and small integrals (Table1 and Figure 3). The rate of increase in NDVI during the beginning of the season can be related to the physiognomy of the vegetation, and the green up rate, as it describes the velocity at which the crop moves from the germination stage to the growth stage. On the other hand, the rate of decrease describes the rate of senescence at which the crop shifts from the flowering phase to the ripening stage, where the harvesting begins. Additionally, the large season integral (LSI) can be used as a proxy of the relative amount of vegetation biomass without regarding the minimum values, whereas the small season integral (SSI) as a proxy of the relative amount of vegetation of biomass while regarding the minimum values ${ }^{29}$. 
Table 1. Explanation of Phenological Metrics ${ }^{32}$

\begin{tabular}{|l|l|}
\hline \multicolumn{1}{|c|}{ Phenological Metric } & \multicolumn{1}{c|}{ Explanation } \\
\hline Time of the start of season (SOS) & $\begin{array}{l}\text { Time for which the value of the index has } \\
\text { increased to a defined level (percentage of } \\
\text { the distance between the left minimum level } \\
\text { and the maximum) }\end{array}$ \\
\hline Time for the peak of the season (POS) & $\begin{array}{l}\text { Computed as the mean value of the times } \\
\text { for which, respectively, the left edge has } \\
\text { increased to the 80\% level and the right } \\
\text { edge has decreased to the 80\% level }\end{array}$ \\
\hline Time of the end of season (EOS) & $\begin{array}{l}\text { Time for which the value of the index has } \\
\text { decreased to a defined level (percentage of } \\
\text { the distance between the right minimum } \\
\text { level and the maximum) }\end{array}$ \\
\hline Base level (BL) & $\begin{array}{l}\text { Given as the average of the left and right } \\
\text { minimum values }\end{array}$ \\
\hline Large seasonal integral (LSI) & $\begin{array}{l}\text { Integral of the function describing the } \\
\text { season from the season start to the season } \\
\text { end }\end{array}$ \\
\hline Small seasonal integral (SSI) & $\begin{array}{l}\text { Integral of the difference between the } \\
\text { function describing the season and the base } \\
\text { level from season start to season end }\end{array}$ \\
\hline
\end{tabular}

The definition of the SOS and the POS can be characterized as crucial, as the left integral defined in combination with the base level, establish a total accumulated biomass indicator of the plant. At the same time, after the finish of the phenological cycle of the crop, the POS and the EOS along with the base level, define a crop production indicator ${ }^{30}$. In addition, high values indicate smaller reduction in the accumulated biomass after the flowering stage of the plant, which are associated with a slower and longer process of producing shoots and fruits, whereas small values represent a shorter process that has not been completed filling shoots and associated with low yield. 


\section{RESULTS}

Using this study's method the start, peak and end DOYs of the crop season have been identified. In addition, based on the extracted SOS and EOS, a biomass and a yield indicator are calculated from the NDVI time-series, using the TIMESAT solution. The biomass indicator can be derived at the peak of the season. Therefore, detecting the onset of the peak of season using appropriate rules, can make pertinent decision making possible, even early in the year.

\subsection{Crop Start and End of Season}

The study of the NDVI profile can identify the three main crop growth phases. After transplanting, which in our case can specify the start of season, the rice crop is at the early vegetative phase, as germination at the seedbeds has proceeded, where the tillering stage begins until the stem elongation of the $\mathrm{crops}^{13}$. Subsequently, the reproductive phase follows, where the peak of season is noted with the highest value of the NDVI time-series, when the crop is at the heading stage ${ }^{32}$. With respect to the end of season, is referred to the DOY where the crop is mature enough to be harvested and thus at the ripening phase.
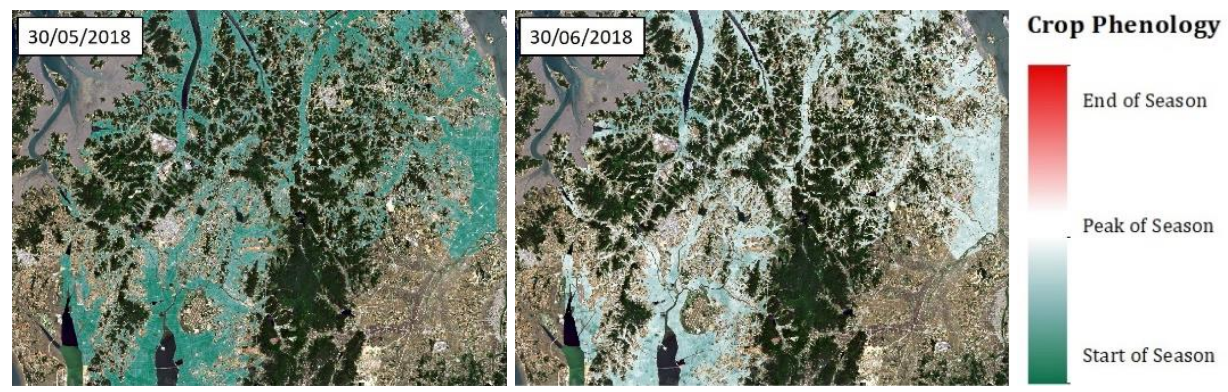

Figure 5. Resulted start of season identifying Vegetative Phase

The study of the NDVI time-series was implemented at the object level, by creating objects of $7 \times 7$ pixels. Figures 5 to 7 illustrate the evolution of rice growth, together with the crop phase. The color pallet, in Figures 5 to 7, illustrates the differences of NDVI values with the peak (highest) NDVI value for every object. 

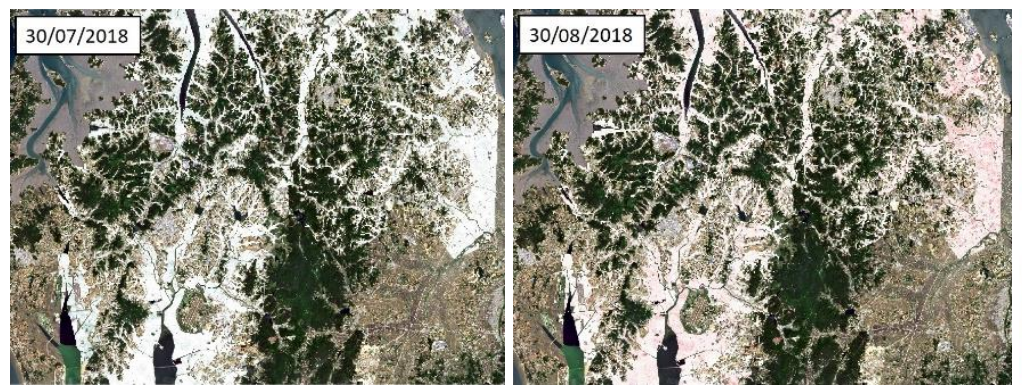

End of Season

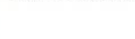

Peak of Season

Start of Season

Figure 6. Resulted peak of season identifying Reproductive Phase

The Figures $5-7$ reveal the development of the rice crop, highlighting with green color the start of season; with white color, the passing to the peak of season; and with red color, when the crop reaches the end of season. The different colors showcase that the rice fields can be at different growth phases at a specific DOY.
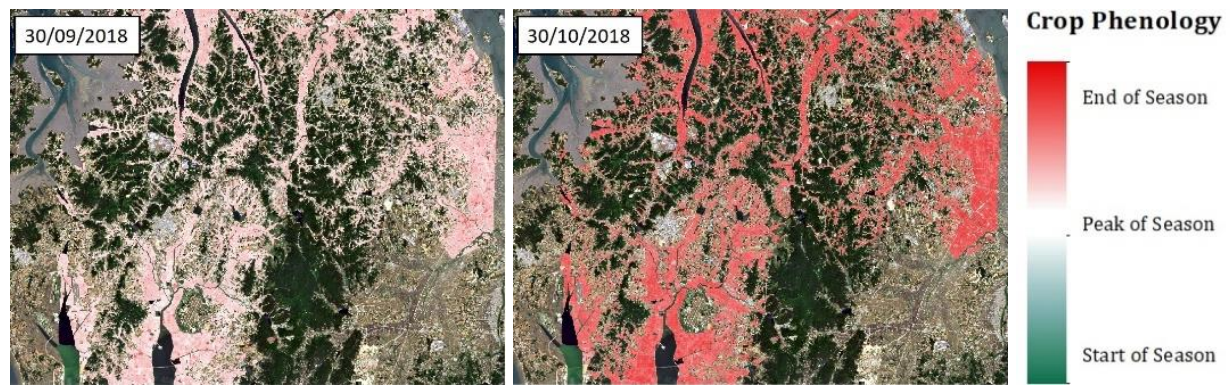

Figure 7. Resulted end of season identifying Ripening phase

\subsection{Phenology Metrics}

As mentioned earlier, the two essential proxies of biomass and yield indicators are obtained from the calculation of start, peak and end of season. These two are produced from the calculation of the left and right integral, between start-peak and peak-end with the base level, respectively. A high value of the biomass indicator implies a high accumulated biomass until the peak of season, which can be used as an early indicator of the final production. 


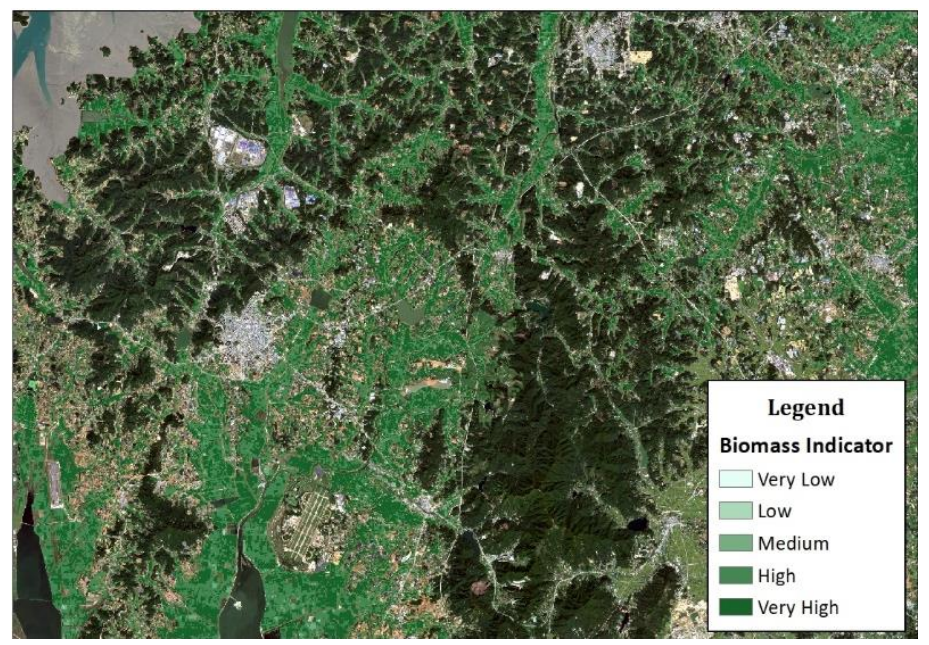

Figure 8. The Biomass Indicator results for the district of Dangjin.

Moreover, at the end of season, the yield indicator is calculated, as a proxy of the final production. The figure of the yield indicator illustrates with deep red color the areas with expectedly high final production. This indicator can be utilized only as a proxy, since it is derived exclusively from the study of the NDVI curve, without considering any other parameters. 


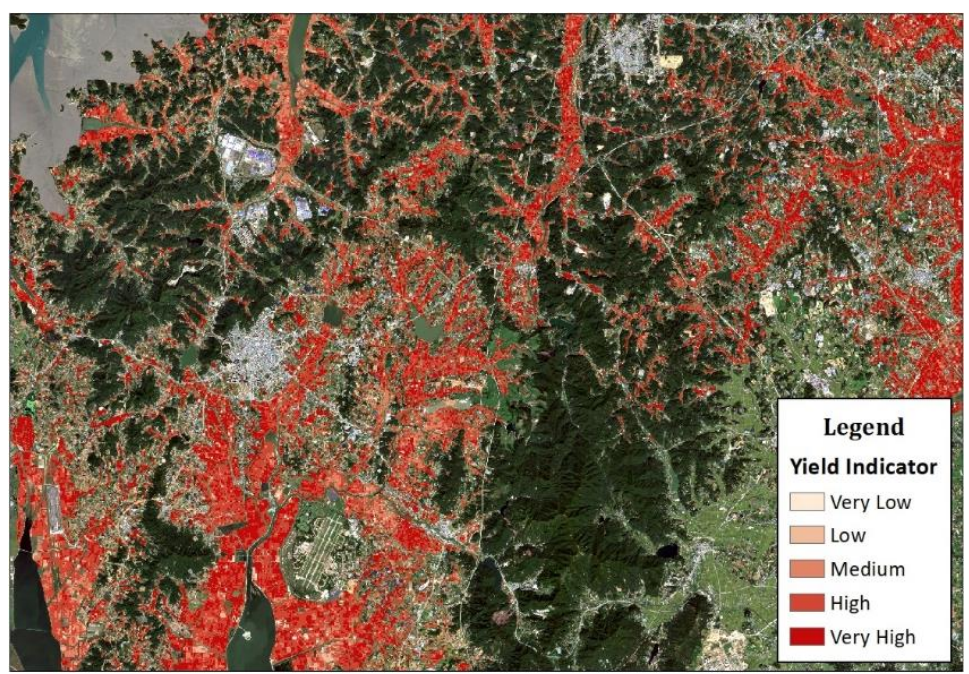

Figure 9. The Yield Indicator results for the district of Dangjin.

\subsection{Validation}

The validation of the rice status indicators was challenging, since no ground truth information was available. The absence of validation crop-calendars for the rice crop at the area of interest, led to the investigation of other means of evaluation. The analysis of the related bibliography provided a benchmark for the primary results of the crop indicators, and that is SOS and EOS. As mentioned by Park et al. (2014) ${ }^{33}$, based on the heading period or the growing days, the rice (Oryza sativa L.), which is cultivated widely in South Korea, can be grouped into early maturing, medium maturing and medium-late-maturing cultivars, which result in different transplanting periods, since the transplanting period is determined by the relative growth difference of rice plants at a certain time. Gutierrez et al. (2013) ${ }^{34}$ highlight that late-maturing fields are flooded twenty-four days more than the early maturing ones. Specifically, as claimed by Jang et al. (2012) $)^{35}$, in the district of Dangjin, mainly early and medium-late maturing rice crops are cultivated. Transplanting starts at the middle of May until the early of June for the medium-late maturing and middle until end of June for the early maturing cultivars. 
With respect to the harvesting practices, Kim et al. (2015) ${ }^{36}$ state that it occurs in October for the medium-late maturing and starts in middle of September for the early maturing cultivars. More than $90 \%$ of the objects were found to have SOS and EOS that fall within the aforementioned periods.
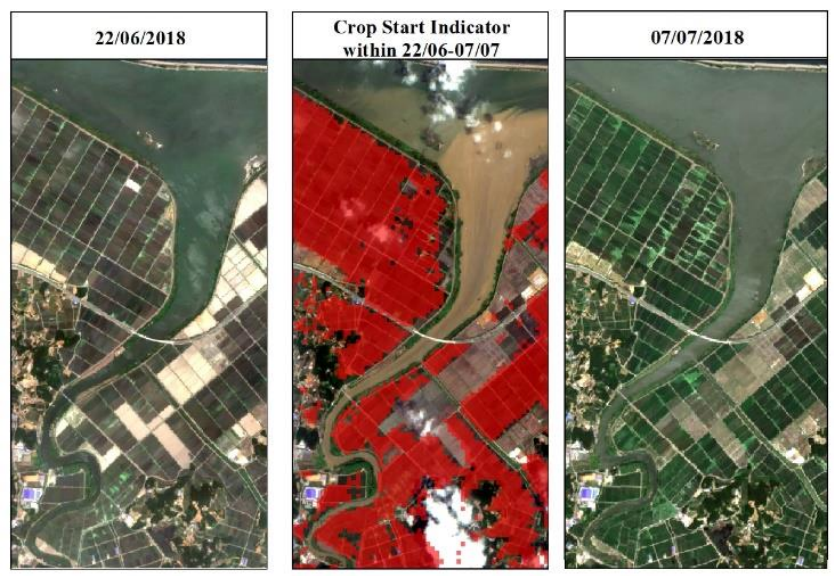

Figure 10. Validation of Crop Start Indicator results through the available cloud free imagery.

Photointerpretation was additionally performed to visually inspect the aforementioned agreement with the periods of transplanting and harvesting mentioned in the literature. Indeed, Figures 10 and 11 reveal that TIMESAT has correctly identified the SOS and EOS timestamps. In Figure 10, it is clearly shown that transplanting occurs sometime between the 22-07/06, while Figure 11 indicates that harvest takes place sometime between 10-15/10. 

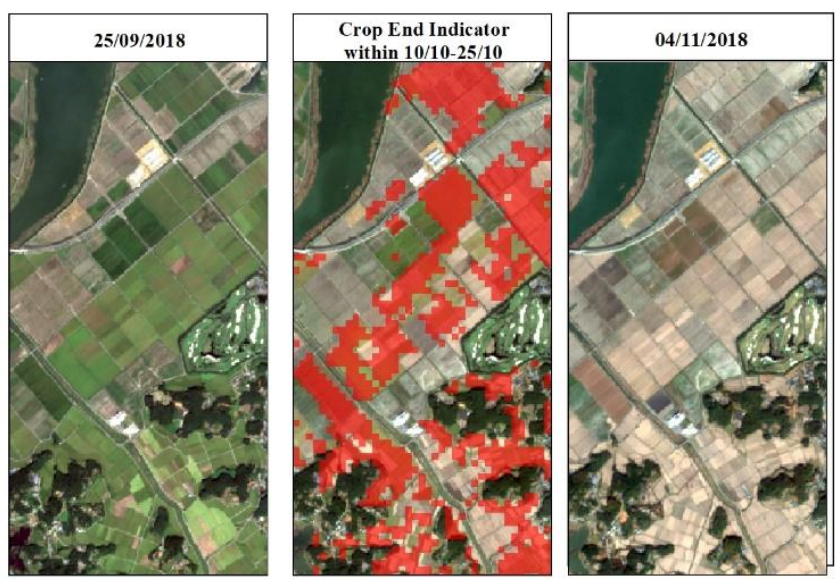

Figure 11. Validation of Crop End Indicator results through the available cloud free imagery.

\section{CONCLUSIONS}

This work, as an extension of the results, described in Sitokonstantinou et al (2019) ${ }^{23}$, adds to an end-to-end Sentinel based agriculture monitoring scheme for the monitoring of food security, but also the control of the new CAP. The suggested scheme describes a transferable and scalable agriculture monitoring system, accommodating for any Big Data considerations. Additionally, the proposed framework addresses the needs of food security and sustainable agriculture stakeholders, such as decision and policy makers, environmental ministries and policy implementation bodies, via providing accurate information, based on which timely actions can be undertaken. In the future, the proposed algorithms will be applied at larger scales, showcasing the scalability of the overall system. At the same time, the scale of thematic information will be increased to the intra-parcel level, incorporating climatic and meteorological data (i.e. accumulated growing degree days) that come from in-situ sensors. 


\section{ACKNOWLEDGEMENTS}

This work was supported by EOPEN project, funded by the European Commission, under the contract number H2020-776019. Authors acknowledge the Copernicus Open Access Hub (https://scihub.copernicus.eu/) for providing free access to Sentinel images. 


\section{REFERENCES}

[1] See, L., Fritz, S., You, L., Ramankutty, N., Herrero, M., Justice, C., Becker-Reshef, I., Thornton, P., Erb, K., Gong, P., Tang, H., van der Velde, M., Ericksen, P., McCallum, I., Kraxner, F. and Obersteiner, M., "Improved global cropland data as an essential ingredient for food security," Global Food Security 4, 37-45 (2015).

[2] Schmedtmann, J. and Campagnolo, M., "Reliable Crop Identification with Satellite Imagery in the Context of Common Agriculture Policy Subsidy Control," Remote Sensing 7(7), 9325-9346 (2015).

[3] "Cross-compliance.", European Commission, 2020, <https://ec.europa.eu/info/foodfarming-fisheries/key-policies/common-agricultural-policy/income-support/crosscompliance_en>.

[4] "Precision agriculture: an opportunity for EU farmers?", European Commission, 18 July 2014, <https://ec.europa.eu/jrc/en/news/precision-agriculture-opportunity-eu-farmers> (2 April 2019).

[5] Kritikos, M., "Precision agriculture in Europe Legal, social and ethical considerations STUDY EPRS | European Parliamentary Research Service Science and Technology Options Assessment" (2017).

[6] Foody, G. M., "Supervised image classification by MLP and RBF neural networks with and without an exhaustively defined set of classes," International Journal of Remote Sensing 25(15), 3091-3104 (2004).

[7] Gu, L., He, F. and Yang, S., "Crop Classification based on Deep Learning in Northeast China using SAR and Optical Imagery," IEEE Xplore, 1 August 2019, <https://ieeexplore.ieee.org/abstract/document/8858437> (27 February 2020 ).

[8] Sitokonstantinou, V., Papoutsis, I., Kontoes, C., Arnal, A., Andrés, A. P. and Zurbano, J. A., "Scalable Parcel-Based Crop Identification Scheme Using Sentinel-2 Data Time-Series for the Monitoring of the Common Agricultural Policy," Remote Sensing 10(6), 911 (2018).

[9] Mazzia, V., Khaliq, A. and Chiaberge, M., "Improvement in Land Cover and Crop Classification based on Temporal Features Learning from Sentinel-2 Data Using Recurrent-Convolutional Neural Network (R-CNN)," Applied Sciences 10(1), 238 (2019).

[10] Porker, K., Eglinton, J., Coventry, S. and Fettell, N., "10 - Improvement of Yield and Adaptation by Manipulating Phenology Genes," ScienceDirect, 1 January 2016, <https://www.sciencedirect.com/science/article/pii/B9780128029220000108>. 
[11]Lambert, M.-J., Traoré, P. C. S., Blaes, X., Baret, P. and Defourny, P., "Estimating smallholder crops production at village level from Sentinel-2 time series in Mali's cotton belt," Remote Sensing of Environment 216, 647-657 (2018).

[12] Skakun, S., Vermote, E., Franch, B., Roger, J.-C., Kussul, N., Ju, J. and Masek, J., "Winter Wheat Yield Assessment from Landsat 8 and Sentinel-2 Data: Incorporating Surface Reflectance, Through Phenological Fitting, into Regression Yield Models," Remote Sensing 11(15), 1768 (2019).

[13] Duchemin, B., Hadria, R., Erraki, S., Boulet, G., Maisongrande, P., Chehbouni, A., Escadafal, R., Ezzahar, J., Hoedjes, J. C. B., Kharrou, M. H., Khabba, S., Mougenot, B., Olioso, A., Rodriguez, J.-C. and Simonneaux, V., "Monitoring wheat phenology and irrigation in Central Morocco: On the use of relationships between evapotranspiration, crops coefficients, leaf area index and remotely-sensed vegetation indices," Agricultural Water Management 79(1), 1-27 (2006).

[14] Jin, X., Kumar, L., Li, Z., Xu, X., Yang, G. and Wang, J., "Estimation of Winter Wheat Biomass and Yield by Combining the AquaCrop Model and Field Hyperspectral Data," Remote Sensing 8(12), 972 (2016).

[15] Jin, X., Li, Z., Yang, G., Yang, H., Feng, H., Xu, X., Wang, J., Li, X. and Luo, J., "Winter wheat yield estimation based on multi-source medium resolution optical and radar imaging data and the AquaCrop model using the particle swarm optimization algorithm," ISPRS Journal of Photogrammetry and Remote Sensing 126, 24-37 (2017).

[16] Silvestro, P. C., Pignatti, S., Pascucci, S., Yang, H., Li, Z., Yang, G., Huang, W. and Casa, R., "Estimating Wheat Yield in China at the Field and District Scale from the Assimilation of Satellite Data into the Aquacrop and Simple Algorithm for Yield (SAFY) Models," Remote Sensing 9(5), 509 (2017).

[17] Ma, G., Huang, J., Wu, W., Fan, J., Zou, J. and Wu, S., "Assimilation of MODIS-LAI into the WOFOST model for forecasting regional winter wheat yield," Mathematical and Computer Modelling 58(3), 634-643 (2013).

[18]Zhou, G., Liu, X. and Liu, M., "Assimilating Remote Sensing Phenological Information into the WOFOST Model for Rice Growth Simulation," Remote Sensing 11(3), 268 (2019).

[19] Fernandes, J. L., Ebecken, N. F. F. and Esquerdo, J. C. D. M., "Sugarcane yield prediction in Brazil using NDVI time series and neural networks ensemble," International Journal of Remote Sensing 38(16), 4631-4644 (2017).

[20] Haghverdi, A., Washington-Allen, R. A. and Leib, B. G., "Prediction of cotton lint yield from phenology of crop indices using artificial neural networks," Computers and Electronics in Agriculture 152, 186-197 (2018). 
[21] Ma, J. W., Nguyen, C. H., Lee, K. and Heo, J., "Convolutional neural networks for rice yield estimation using MODIS and weather data: A case study for South Korea," Journal of the Korean Society of Surveying, Geodesy, Photogrammetry and Cartography 34(5), 525-534 (2016).

[22] Yalcin, H., "Plant phenology recognition using deep learning: Deep-Pheno," IEEE Xplore, 1 August 2017, <https://ieeexplore.ieee.org/document/8046996? section=abstract> (3 March 2020 ).

[23] Sitokonstantinou, V., Drivas, T., Koukos, A., Papoutsis, I. and Kontoes, C., "SCALABLE DISTRIBUTED RANDOM FOREST CLASSIFICATION FOR PADDY RICE MAPPING," zenodo.org, $11 \quad$ February <https://zenodo.org/record/3662151\#.XnjYw4gzaUk> (23 March 2020 ).

[24] Park, S., Im, J., Park, S., Yoo, C., Han, H. and Rhee, J., "Classification and Mapping of Paddy Rice by Combining Landsat and SAR Time Series Data," Remote Sensing 10(3), 447 (2018).

[25] Kim, M., Ko, J., Jeong, S., Yeom, J. and Kim, H., "Monitoring canopy growth and grain yield of paddy rice in South Korea by using the GRAMI model and high spatial resolution imagery," GIScience \& Remote Sensing 54(4), 534-551 (2017).

[26] Lebourgeois, V., Dupuy, S., Vintrou, É., Ameline, M., Butler, S. and Bégué, A., "A Combined Random Forest and OBIA Classification Scheme for Mapping Smallholder Agriculture at Different Nomenclature Levels Using Multisource Data (Simulated Sentinel-2 Time Series, VHRS and DEM)," Remote Sensing 9(3), 259 (2017).

[27] Eklundh, L. and Jönsson, P., "TIMESAT : A Software Package for Time-Series Processing and Assessment of Vegetation Dynamics," muep.mau.se, 2015, <http://muep.mau.se/handle/2043/20074> (23 March 2020).

[28] Penatti, N. C. and de Almeida, T. I. R., "SUBDIVISION OF PANTANAL QUATERNARY WETLANDS: MODIS NDVI TIMESERIES IN THE INDIRECT DETECTION OF SEDIMENTS GRANULOMETRY,” ISPRS - International Archives of the Photogrammetry, Remote Sensing and Spatial Information Sciences, 311-316 (2012).Hanhee, H. and Ham, H.-H., "Rice and Koreans: Three Identities and Meanings," Korea Journal, 89-106 (2005).

[29] Jönsson, P. and Eklundh, L., "TIMESAT — a program for analyzing time-series of satellite sensor data," Computers \& Geosciences 30(8), 833-845 (2004).

[30] Araya, S., Ostendorf, B., Lyle, G. and Lewis, M., "CropPhenology: An R package for extracting crop phenology from time series remotely sensed vegetation index imagery," Ecological Informatics 46, 45-56 (2018). 
[31] JIN, C., XIAO, X., DONG, J., QIN, Y. and WANG, Z., "Mapping paddy rice distribution using multi-temporal Landsat imagery in the Sanjiang Plain, northeast China," Frontiers of earth science 10(1), 49-62 (2016).

[32] Parul, S., "Rice technical manual for extension officers," Food and Agriculture Organization of the United Nations, Apia, 3-6 (2017).

[33] Li, F. P., Lee, Y. S., Kwon, S. W., Li, G. and Park, Y. J., "Analysis of genetic diversity and trait correlations among Korean landrace rice (Oryza sativa L.)," Genetics and Molecular Research 13(3), 6316-6331 (2014).

[34] Gutierrez, J., Kim, S. Y. and Kim, P. J., "Effect of rice cultivar on CH4 emissions and productivity in Korean paddy soil," Field Crops Research 146, 16-24 (2013).

[35] Min-Won Jang, Kim, Y.-H., No-Wook Park and Hong, S.-Y., "Mapping Paddy Rice Varieties Using Multi-temporal RADARSAT SAR Images," 2012, <https://www.semanticscholar.org/paper/Mapping-Paddy-Rice-Varieties-Using-Multitemporal-Jang-Kim/2b946e9870ad2bec94f176408d9036551d220b95> (24 March 2020 ).

[36] Kim, H.-O. and Yeom, J.-M., "Sensitivity of vegetation indices to spatial degradation of RapidEye imagery for paddy rice detection: a case study of South Korea," GIScience \& remote sensing (2015). 